\title{
Enhanced precipitate growth at reduced temperature during chemical ordering in deformed red gold
}

\section{alloys}

Marina Garcia-Gonzalez ${ }^{\mathrm{a}, \mathrm{b}}$, Steven Van Petegem ${ }^{\mathrm{a}}$, Nadine Baluc ${ }^{\mathrm{c}}$, Samy Hocine ${ }^{\mathrm{a}, \mathrm{b}}$, Maxime Dupraz ${ }^{\mathrm{a}, \mathrm{b}}$, Fanny Lalire ${ }^{\mathrm{d}}$, Helena Van Swygenhoven ${ }^{\mathrm{a}, \mathrm{b}, *}$

${ }^{\mathrm{a}}$ Photons for Engineering and Manufacturing, Paul Scherrer Institut,5232 Villigen PSI, Switzerland

${ }^{b}$ Neutrons and X-Rays for Mechanics of Materials, École Polytechnique Fédérale de Lausanne, 1015 Lausanne, Switzerland

${ }^{c}$ Centre Interdiscipinaire de Microscopie Electronique, Ecole Polytechnique Fédérale de Lausanne, 1015 Lausanne, Switzerland

${ }^{\mathrm{d}}$ VARINOR S.A., 2800 Delémont, Switzerland

*Corresponding Author

Prof. Helena Van Swygenhoven

Paul Scherrer Institut

CH-5322 Villigen PSI, Switzerland

helena.vs@psi.ch

\section{ABSTRACT}

Manufacturing of red gold components relies widely on controlling the formation of ordered precipitates during thermo-mechanical processing.The ordered phase hardens the material, makes it less workable and introduces residual stresses. We show that an industrial wire straightening process on chemically disordered wires enhances the formation of ordered precipitates in the near surface region during natural ageing. To address the role of plastic deformation during artificial ageing, in situ X-ray experiments are carried out on deformed and undeformed samples. It is shown that pre-deformation decreases the onset temperature for ordering and enhances precipitation growth at the early stages of the transformation.

Keywords: X-ray diffraction; Gold alloys; Ordering; Plastic Deformation; Ageing

This document is the accepted manuscript version of the following article:

Garcia-Gonzalez, M., Van Petegem, S., Baluc, N., Hocine, S., Dupraz, M., Lalire, F., \& Van Swygenhoven, H. (2019). Enhanced precipitate growth at reduced temperatures during chemical ordering in deformed red gold alloys. Scripta Materialia, 170, 129-133.

https://doi .org/10.1016/j.scriptamat. 2019.05.038

This manuscript version is made available under the CC-BY-NC-ND 4.0 1icense http:// creativecommons.org/1icenses/by-nc-nd/4.0/ 
The manufacturing of 18-carat red gold components (nominal composition Au-43.3at.\%Cu-5.6at.\%Ag) relies widely on controlling precipitation hardening. This alloy has a chemical composition close to equiatomic AuCu and undergoes the same disorder-order transition from a chemically disordered fcc A1 (space group Fm3m) to an ordered tetragonal L10 (space group P4/mmm) [1,2]. Ordering in AuCu-based alloys can be suppressed by rapidly cooling down after recrystallization heat treatments i.e by freezing in the metastable disordered state. However, coherent precipitates start forming already at room temperature, a process in which the quenched-in excess vacancies play a role [3]. The tetragonal lattice distortion introduces at the early stages of the transformation misfit strains in the matrix which upon further growth of the precipitates can be relieved by twinning, fracture or spontaneous recrystallization[4-6]. The A1 phase is ductile and workable allowing easy manufacturing while the L10 phase is harder but brittle [7]. Microalloying is a strategy that is exploited in the precious metal and dental industry to reduce shape distortions after age hardening of components[8,9]. The addition of silver reduces the critical temperature for ordering[10], delays the ordering kinetics [11] and retards the growth rates of the inter-twin spacing and antiphase domain size [12]. Red gold undergoes the order-disorder transformation below $360^{\circ} \mathrm{C}$ and is subject to shape distortions during processing as opposed to similar alloys with lower amount of copper. While many studies focused on the age-hardening behaviour and microstructure development after ageing [1218], very few focus on the role of plastic deformation at the early stages of ordering.

It is very well known that the strain field of a dislocation facilitates the nucleation of precipitates [19,20]. Possible mechanisms are stress induced local changes in lattice parameter, which accommodate the precipitate more readily; or locally induced chemical composition changes that reduce the critical ordering temperature[21]. It is however not clear if and how dislocations influence the degree of ordering during ageing as some of the reported results are contradictive. Quenched $\mathrm{AuCu}$ was found to age harden more during annealing at $150^{\circ} \mathrm{C}$ than a previously deformed sample, the differences decreased when annealing at $240^{\circ} \mathrm{C}$ [6]. A TEM study showed that the degree of ordering after isothermal annealing of a deformed sample is less than that in the non-deformed sample when annealing is performed below $330^{\circ} \mathrm{C}$. The opposite is true when annealing is performed close to the critical temperature for ordering [22]. Resistivity measurements during heating of quenched AuCu samples suggested a delay in ordering in samples that were previously deformed [23]. The results are explained by a competing role played by ordering, the dislocation network and the recrystallizing microstructure. However, a fundamental understanding of these phenomena is missing.

In this letter we first show that the degree of ordering is higher in surface regions when a red gold wire is straightened after recrystallization in an industrial continuous furnace. To address the role of dislocations during artificial ageing, in situ X-ray experiments are carried out on deformed and non-deformed samples, and complemented with TEM observations. It is shown that the presence of dislocations accelerates the ordering kinetics and enhances precipitation growth, which starts at a lower temperature.

Samples with nominal composition of Au-20.5wt.\%Cu-4.5wt.\%Ag were prepared at VARINOR S.A. by cast melting from high purity Au, Cu and Ag under a high-purity argon atmosphere. The specimens underwent a series of drawing operations followed by recrystallization until wires with a cross-section of $2 \times 4 \mathrm{~mm}^{2}$ were obtained. After a final recrystallization step at $660^{\circ} \mathrm{C}$ four wires were prepared for the $e x$ situ test (Table 1) using two different cooling rates $\left(-800^{\circ} \mathrm{C} / \mathrm{min}\right.$ and $\left.-100^{\circ} \mathrm{C} / \mathrm{min}\right)$ in order to vary the initial fraction of ordered phase. After cooling, the wire is slightly distorted and has a wavy character. Two of the wires were straightened in a wire-straightening machine. All samples were exposed to natural ageing for 7 days. Finally, thin slices $(\sim 0.3 \mathrm{~mm})$ are cut from the wires. For the in situ studies four $2 \times 4 \mathrm{~mm}^{2}$ samples $(550 \mathrm{R}-$ 
s) were recrystallized at $550^{\circ} \mathrm{C}$ and cooled down to room temperature with a cooling rate of $-800^{\circ} \mathrm{C} / \mathrm{min}$. The lower recrystallization temperature prevents excessive grain growth. The grain size of the samples was determined by optical microscopy (samples 660R-s and 660Rf) and electron backscatter diffraction (EBSD) (550R-s). An EBSD map of the latter is provided in Fig. S1 of Supplementary Material. Electron microscopy (TEM) observations were performed in a FEI TALOS F200S operating at 200keV. Samples were prepared as 3mm discs, manually polished and further thinned by double-jet electropolishing in a TENUPOL 5. The electrolyte’s solution contained 50\% HCl : 35\%glycerol : $15 \%$ ethanol in volume percent.

The X-ray diffraction experiments are performed at the High Energy Materials Science Beamline (P07) at Petra III [24]. A schematic view of the setup is shown in Fig. 1a. The X-ray beam had an energy of $77.7 \mathrm{keV}$ and a size of $0.2 \times 0.2 \mathrm{~mm}^{2}$. Diffracted X-rays are recorded with a Perkin Elmer XRD 1621 Flat Panel detector, which is positioned at a distance of 1.4m. Exposure times were $0.1 \mathrm{~s}$ and $1 \mathrm{~s}$ for the in and ex situ measurements, respectively. Calibration of the setup was performed using a standard $\mathrm{CeO}_{2}$ powder yielding precise information on the position of the beam centre, the sample-to-detector distance and the detector tilt.

\section{Table 1}

Overview of processing parameters for ex situ samples $\left(\mathrm{C}_{\mathrm{r}}=\right.$ cooling rate $)$. The nomenclature is defined as follows: $\mathrm{xxxR}=$ recrystallization temperature, $\mathrm{S}=$ straightened, $\mathrm{f}, \mathrm{s}=$ fast or slow cooling.

\begin{tabular}{lcccc}
\hline Nomenclature & $\begin{array}{c}\mathrm{T}_{\mathrm{r}} \\
\left({ }^{\circ} \mathrm{C}\right)\end{array}$ & $\begin{array}{c}\mathrm{C}_{\mathrm{r}} \\
\left({ }^{\circ} \mathrm{C} / \mathrm{min}\right)\end{array}$ & Straightened & $\begin{array}{c}\text { Grain size } \\
\text { 660R-f }\end{array}$ \\
\hline 660 & -800 & - & $13 \mathrm{~m})$ \\
660R-s & 660 & -100 & - & 30 \\
660RS-f & 660 & -800 & 0 & 13 \\
660RS-s & 660 & -100 & 0 & 30 \\
550R-s & 550 & -800 & - & 8 \\
\hline
\end{tabular}

For the in situ measurements the samples are mounted in a custom-built heating chamber, which in turn is mounted on a heavy load PI hexapod. Fig. 1b displays a schematic view of the heating chamber. It is composed of two 90W silicon nitride ceramic heating elements (BACH Resistor Ceramics $\mathrm{GmbH}$ ) and a copper sample holder within an aluminium box. With the aid of finite element simulations using the ABAQUS/standard software [25] the sample holder was designed such that it provides a homogeneous temperature distribution in the centre of the samples and attains cooling rates as fast as $-800^{\circ} \mathrm{C} / \mathrm{min}$ in a controlled way. A thin $0.3 \mathrm{~mm}$ sample is clamped between two copper pieces with an $\mathrm{X}$-ray entrance and exit opening. The temperature is measured by a fine gauge K-type thermocouple (OMEGA GmbH), clamped between the sample and the sample holder. Controlled cooling is achieved by a reduction of the current through the heating elements, combined with blowing argon gas directly on the sample.

For the ex situ measurements the samples are mounted side-by-side on a sample holder on top of the heating chamber. For each sample a line scan across the thickness of the sample was performed resulting in 20 diffraction patterns recorded at mid-thickness along the $2 \mathrm{~mm}$ width, as illustrated by the yellow line in Fig. 1c. Each point is separated by $0.1 \mathrm{~mm}$. 
a)

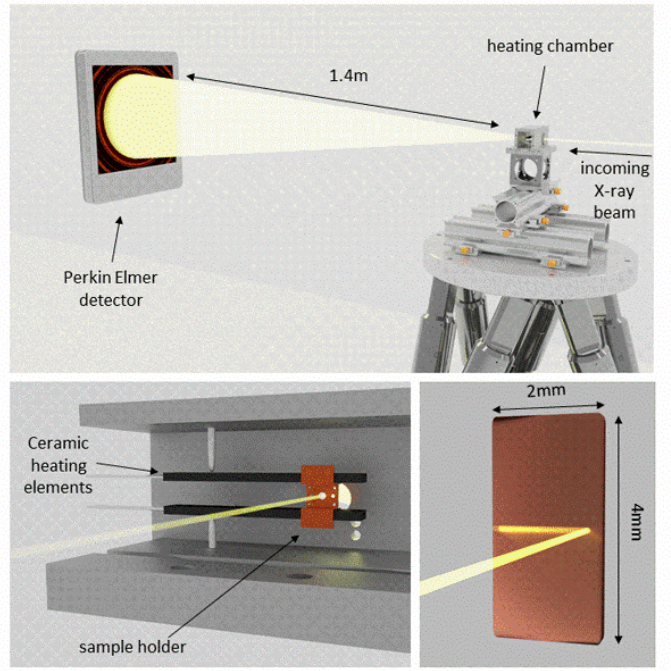

b)

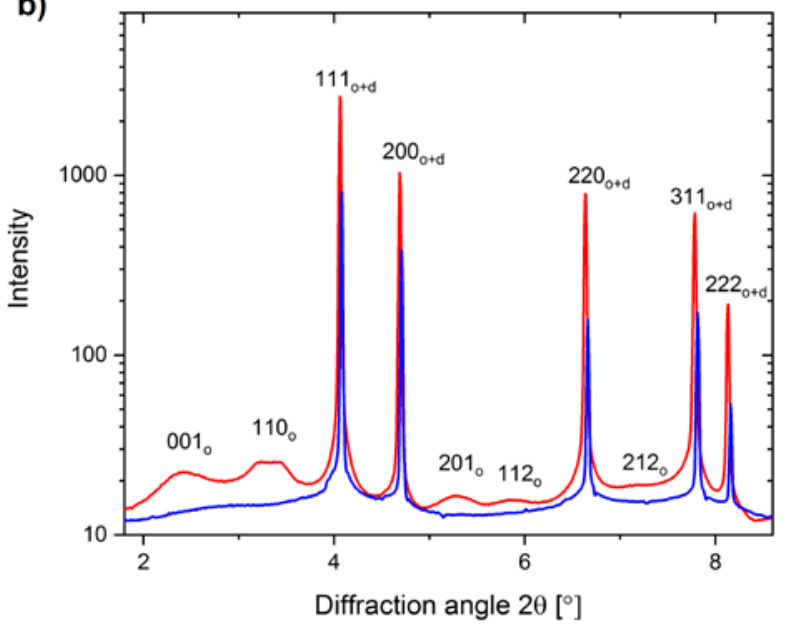

Fig 1. a) Schematic view of the in situ X-ray diffraction setup at the P07 beam line, b) Part of the heating chamber showing the Cu sample holder mounted between two ceramic heaters, c) scanning procedure applied for the ex situ experiments, d) Representative $1 \mathrm{D}$ diffraction patterns recorded at $660^{\circ} \mathrm{C}$ (blue curve) and at room temperature after ageing (red curve) showing diffraction peaks from both the ordered (o) and disordered (d) phase.

The 2D X-ray diffraction patterns are reduced to conventional 1D patterns by azimuthal integration using the DAWN software package [26].

Fig. 1d displays representative diffraction patterns in the disordered state (at $660^{\circ} \mathrm{C}$ ) and a partly ordered state at room temperature. The lattice parameters of the disordered A1 and the ordered L10 phase are very similar, resulting in strongly overlapping fundamental peaks.In the early stages of ordering the nanoprecipitates are coherent with the parent matrix, inducing short range order and misfit strains. Short range order together with elevated temperature result in strong diffuse scattering components[27-29]. State-of-the-art full pattern fitting methods cannot account for a combination of these features. Therefore, single peak fitting with PearsonVII functions was performed using in-house written Matlab routines. 


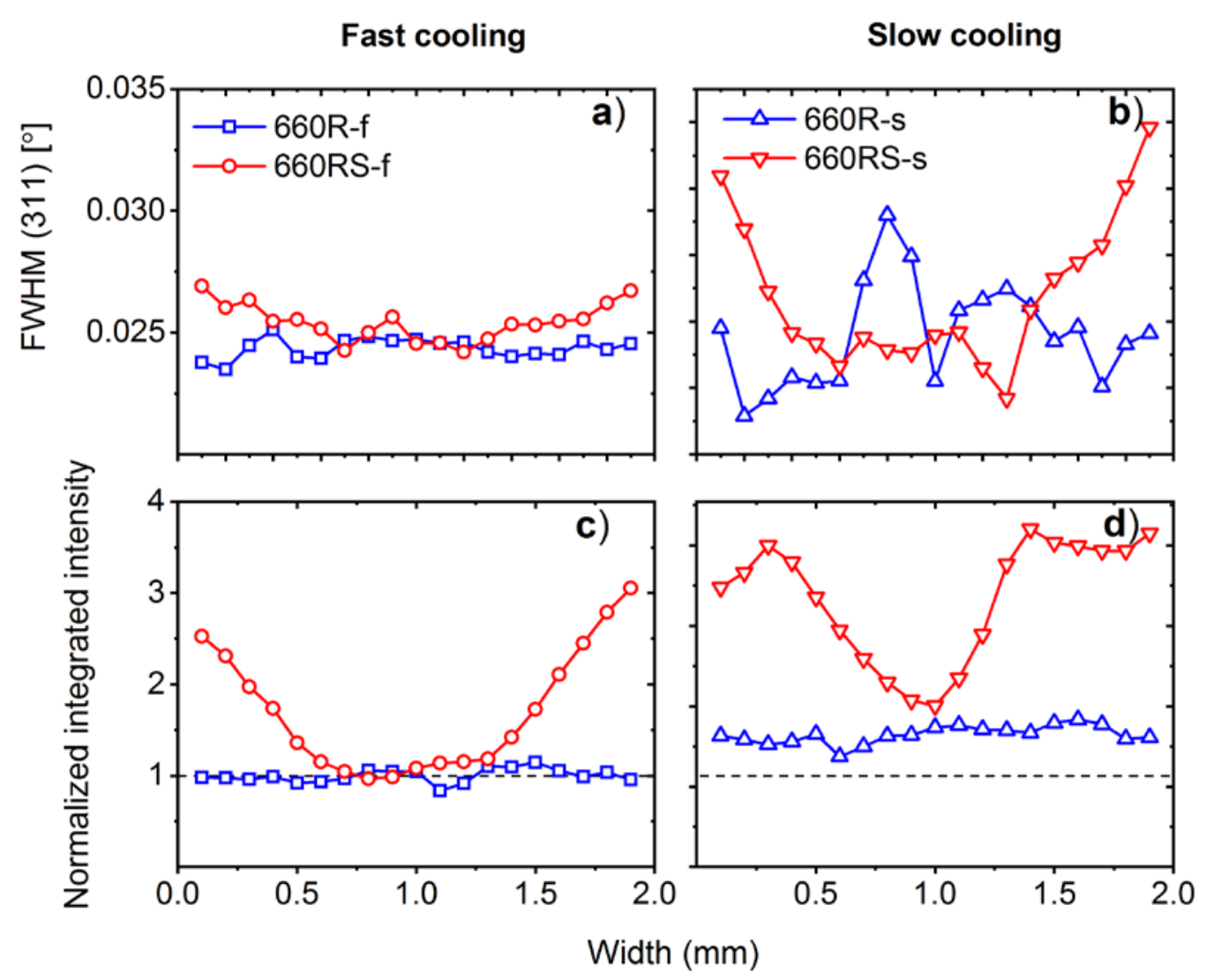

Fig 2. Peak broadening of the (311) diffraction peak (a-b) and integrated intensity of the (110) diffraction peak (c-d) across the width of the samples

Plastic deformation induced by the wire straightening operation increases the peak broadening of all fundamental diffraction peaks, as illustrated for the (311) fundamental peak in Fig. 2a-b for the line scans performed on the samples listed in table 1. The alloy grain size is too large to contribute to the peak broadening. It should be noted that the (311) peak might comprise contributions of both phases. However, the intensity of the ordered phase is very small and can therefore be ignored. As a result, we can assume that the peak broadening is directly related to inhomogeneous elastic strains in the disordered matrix.

For the non-straightened samples the FWHM of (311) is expected to be independent on the position within the samples. This is the case of 660R-f. The FWHM for 660R-s exhibits a lot of scatter, which can be ascribed to a lack in grain statistics. This sample had been exposed longer to high temperature then 660R-f, leading to larger grain sizes. This is also confirmed by the 2D patterns of 660R-s, which do not exhibit full diffraction rings from the disordered phase; in contrast, they are rather spotty and the intensities vary strongly when scanning across the thickness (see Fig. S2 in the Supplementary Material). The rings of the superlattice peaks however were continuous in all samples. The straightening is reflected by a small increase in the FWHM near the surface in all samples.

Figs. 2c-d compare the integrated intensities of the (110) superlattice reflection of the L10 phase. Similar profiles are obtained for other superlattice reflections. The intensities are normalized to the average value for sample 660R-f. The recrystallized samples without straightening (660R-s,f) show as expected the presence of homogeneously distributed ordered precipitates. The averaged intensity is slightly higher in 660Rs then in 660R-f. After straightening, the integrated intensity is clearly higher near the surface of the samples, suggesting differences in 
distribution and/or sizes of ordered precipitates. The profiles are quite different for fast and slow cooling rates. Where there is a clear correlation between the integrated intensity and the FWHM for the fast cooled samples, this is not the case for the slowly cooled samples. Here, the maximum integrated intensity is reached below the surface. Since the industrial wire straightening process is carried out on a wavy wire, varying levels of plastic deformation along the length of the wire are to be expected and therefore, the exact shape of the profiles shown in Fig. 3c and 3d may not be directly comparable.
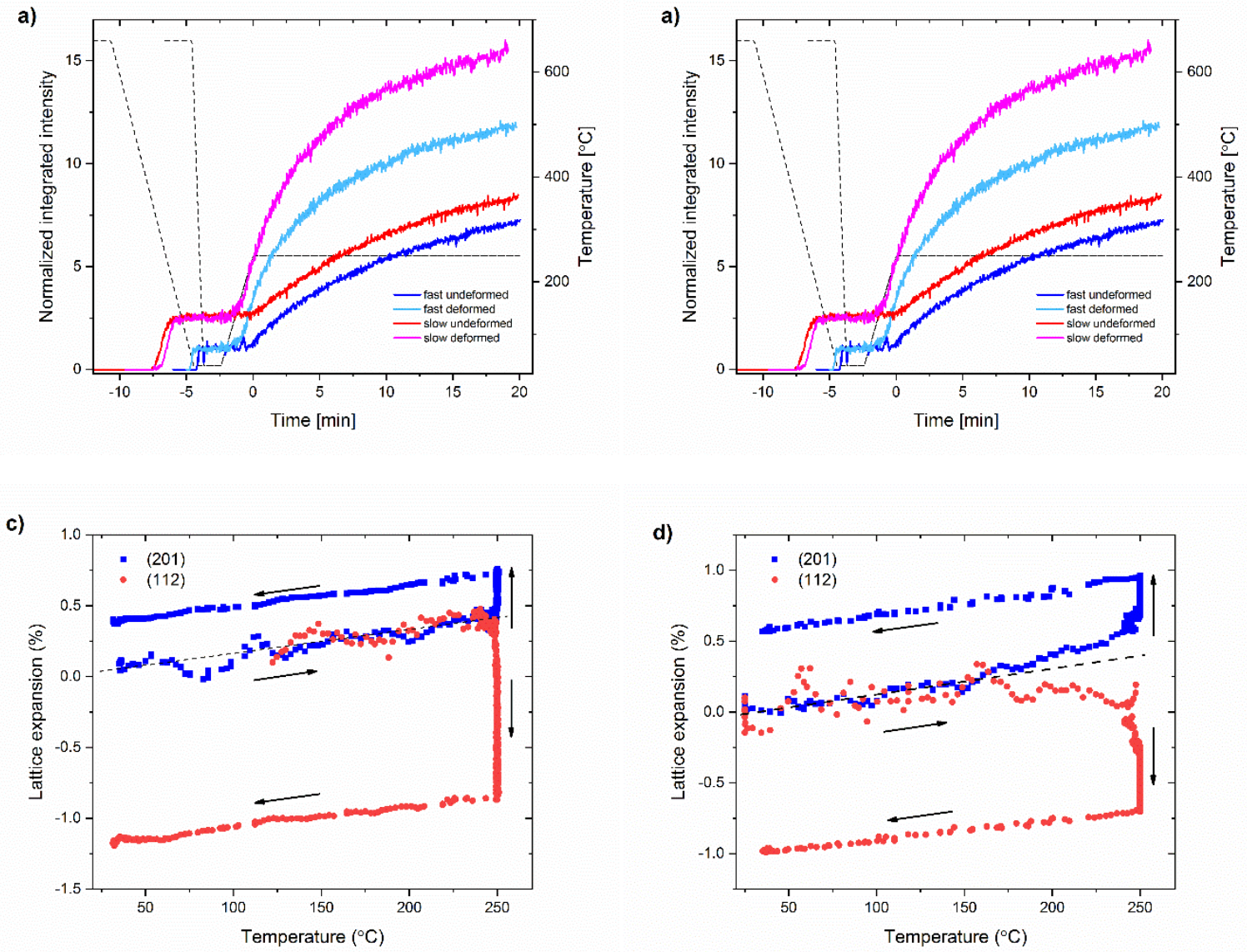

Fig 3. a-b) Evolution of the integrated intensity of the (110) diffraction peak during a thermal cycle, which is comprised of slow or fast cooling from $660^{\circ} \mathrm{C}$ followed by aging at $250^{\circ} \mathrm{C}$ with and without mechanical deformation in between. a) shows the temperature profile (dashed lines) and integrated intensities (full lines) as a function of time, b) displays the integrated intensity during heating up to the ageing temperature $\left(250^{\circ} \mathrm{C}\right)$. c) Evolution of the peak position of the (201) and (112) diffraction peaks during the thermal cycle in the undeformed sample (slowly cooled). d) Evolution of the peak position of the (201) and (112) diffraction peaks during thermal cycle in the deformed sample (slowly cooled). The black arrows indicate the heating and cooling sections.

To verify whether dislocations also play a role in enhancing ordering during artificial ageing, a treatment frequently applied during processing of precious metal objects, four in situ diffraction experiments were performed. First, all 4 samples are heated to $660^{\circ} \mathrm{C}$ with a fixed heating rate of $100^{\circ} \mathrm{C} / \mathrm{min}$ and kept there for $2 \mathrm{~min}$ to ensure a complete dissolution of the L10 precipitates, followed by controlled cooling to room temperature with cooling rates of $-100^{\circ} \mathrm{C} / \mathrm{min}$ and $-800^{\circ} \mathrm{C} / \mathrm{min}$. Two of the samples were then cold rolled to a thickness reduction of $25 \%$ using a Cooks rolling mill 999 AXWA. Finally, all 4 samples are subjected to a $20 \mathrm{~min}$ ageing treatment at $250^{\circ} \mathrm{C}$. It should be noted that the cold rolling process induces a much higher degree of plastic deformation as compared to the wire straightening process. This is evidences in Fig. S3 in Supplementary Material, which compares the changes of the FWHM of the (311) diffraction peak between the cold rolling and wire 
straightening process. Fig. 3a shows the evolution of the integrated intensity of the (110) superlattice reflection together with the temperature profiles (dashed black lines) starting from the recrystallization temperature $\left(660^{\circ} \mathrm{C}\right)$. The intensities are normalized to the intensity obtained at room temperature after fast cooling. During cooling, precipitates start forming below $360^{\circ} \mathrm{C}$. Below $200^{\circ} \mathrm{C}$ the ordering kinetics slows down and a plateau is reached. At room temperature, the integrated intensity after slow cooling is more than twice that after fast cooling. After room temperature is reached, the temperature is raised again to the ageing temperature at $250^{\circ} \mathrm{C}$. During this period, the integrated intensity raises much faster in the deformed samples than in the undeformed samples. In the deformed samples ordering becomes visible around $100^{\circ} \mathrm{C}$ whereas in the non-deformed samples a raise is only observed close to $250^{\circ} \mathrm{C}$. This is evidenced in Fig. 3b, showing the evolution of the integrated intensities during heating from room temperature to the ageing temperature $\left(250^{\circ} \mathrm{C}\right)$. After the ageing temperature of $250^{\circ} \mathrm{C}$ is reached (corresponding to time zero in Fig 3a), the difference between the integrated intensity of the deformed and the undeformed sample further increases. However towards the end of the ageing, the difference seems to remain constant. In other words, cold rolling prior to ageing increases the rate of ordering at the early stages

The integrated intensity is proportional to the volume fraction of the precipitates and therefore cannot discriminate between growth or nucleation. Further information can be obtained by considering the evolution of elastic strain in the precipitates. Fig. 3c and 3d show the evolution of the change of the interplanar spacing of the (201) and (112) superlattice reflection relative to their value prior to heating for the slowly cooled undeformed and deformed sample, respectively. Fig. S4 in Supplementary Material shows the corresponding evolution as a function of time. During initial heating to the ageing temperature the lattice expands, thereby increasing the interplanar spacing. From this we can derive a thermal expansion coefficient of $16 \times 10^{-6} \mathrm{C}^{-1}$, in agreement with the value reported in [30]. The same value is obtained during cooling down after 20 min ageing. After reaching the ageing temperature of $250^{\circ} \mathrm{C}$ the interplanar spacings of the (201) and (112) peak of the undeformed sample change significantly and in opposite direction. For the deformed sample deviation from thermal expansion starts already at $150^{\circ} \mathrm{C}$. It is well known that during early stages of ordering the precipitates are fully coherent with the matrix and are under tension along the c-axis and compressed along the a-axes [1,5]. During precipitate growth the unit cell parameters approach those of the constraint-free AuCu-I phase [1]. This results in an expansion and contraction of the (201) and (122) interplanar spacings, respectively. With other words, the evolution of lattice expansion in Fig. 3c and 3d demonstrate that in the deformed sample the precipitates start to grow at a lower temperature as compared to the undeformed sample.

Post-mortem TEM studies reveal different nano-precipitate microstructures in the undeformed and deformed samples. Figs. 4a and $4 \mathrm{~b}$ show dark field TEM images based on the (1-10) superlattice reflection along the $<110>$ zone axis of respectively the undeformed and deformed sample after ageing. Fig. S5 in Supplementary Material shows the corresponding diffraction patterns. The undeformed sample exhibits a very fine dispersion of homogeneously distributed precipitates, whereas in the predeformed sample the precipitates are slightly coarser and heterogeneously distributed. Micro-diffraction performed in the dark areas of the deformed samples shows no superlattice reflections. Unfortunately, it was not possible to observe simultaneously dislocations and precipitates because for the latter, the film thickness has to be below 100nm. . Additionally, the abscence of twinning or antiphase boundaries in the microstructure, characteristic coherency strain relief mechanisms [1], confirms that the early stages of ordering prevail and that precipitates are coherent with the matrix. 
a)

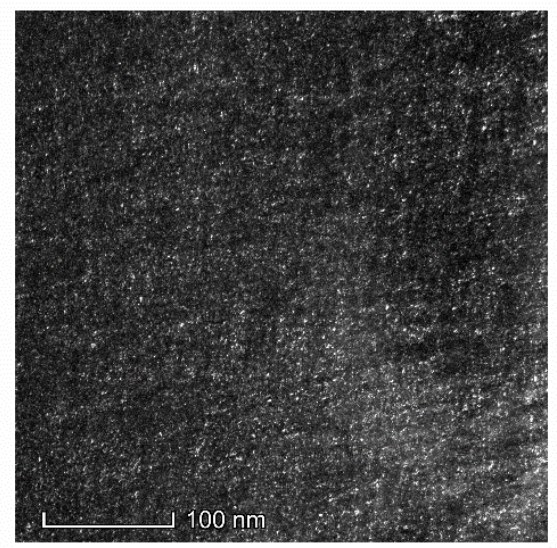

b)

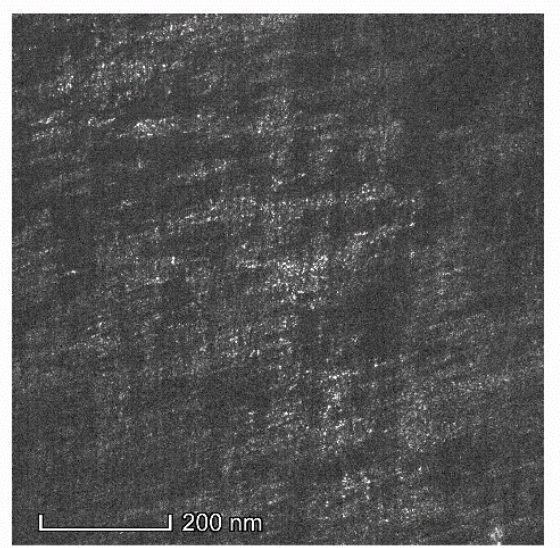

Fig. 4. a-b) Dark field TEM images based on the (1-11) super-lattice reflection along the $<110>$ zone axis after 20min ageing, a) without and b) with prior deformation.

In summary, plastic deformation enhances the ordering kinetics at RT and during artificial ageing, two thermal treatments where the relative importance of parameters affecting ordering, such as quenched-in excess vacancies, interdiffusion of silver and copper and strain field around a dislocation, are different. Together with the heterogeneous distribution of precipitates observed in TEM in the deformed samples, our experiments show that dislocations in red gold facilitate ordering. We anticipate that dedicated in situ heating experiments can provide activation energies allowing pinning down the role of vacancies and dislocations on the ordering kinetics.

\section{Acknowledgements}

We acknowledge DESY (Hamburg, Germany), a member of the Helmholtz Association HGF, for the provision of experimental facilities. This research was carried out at PETRA III in the P07 beam line with the support of Dr. Torben Fischer.

\section{References}

[1] M. Hirabayashi, S. Weissmann, Acta Metall. 10 (1962) 25-36.

[2] K. Udoh, M. Ohta, K. Oki, and K. Hisatsune, (2000).

[3] K. Hisatsune, Y. Tanaka, K. Udoh, K. Yasuda, Intermetallics 3 (1994) 335-339.

[4] S. Kohara, G.C. Kuczynski, Acta Metall. 4 (1956) 221-222.

[5] G.V. Tendeloo, S. Amelinckx, S.J. Jeng, C.M. Wayman, J. Mater. Sci. 21 (1986) 4395-4402.

[6] V. S. Arunachalam, R. W. Cahn, J. Mater. Sci. 2 (1967) 160-170.

[7] Spanl, M, Puschl W., Sprusil, B., Sachl J., Sima, V., Pfeiler, W., Mater. Trans. 43 (2002) 560-565.

[8] M. Ohta, T. Shiraishi, R. Ouchida, M. Nakagawa, S. Matsuya, J. Alloys Compd. 265 (1998) 240-248.

[9] I. Watanabe, M. Atsuta, K. Yasuda, K. Hisatsune, Dent. Mater. J. 10 (1994) 369-374.

[10] L. Amiour, S. Mermoul, D. Hamana, Phys. Procedia 55 (2014) 30-34.

[11] H.K. K. Yasuda, Bull Tokyo Med Dent Univ 22 (1975) 101-111.

[12] T. Shiraishi, M. Ohta, M. Nakagawa, R. Ouchida, J. Alloys Compd. 257 (1997) 306-312.

[13] M. Ohta, T. Shiraishi, M. Nakagawa, S. Matsuya, J. Mater. Sci. 29 (1994) 2083-2086.

[14] M. Ohta, T. Shiraishi, M. Yamane, K. Yasuda, Dent. Mater. J. 2 (1983) 10-17.

[15] L. Pezzato, G. Magnabosco, K. Brunelli, M. Breda, M. Dabalà, Metallogr. Microstruct. Anal. 5 (2016) 116-123.

[16] Y. Kim, M. Niinomi, M. Nakai, T. Akahori, T. Kanno, H. Fukui, J. Alloys Compd. 519 (2012) 15-24.

[17] H-I. Kim, D-H. Lee, J-A. Sim, Y-H. Kwon, H-J. Seol, Mater. Charact. 60 (2009) 357-362.

[18] R. Ouchida, T. Shiraishi, M. Nakagawa, M. Ohta, J. Mater. Sci. 30 (1995) 3863-3866.

[19] W.A. Curtin, D.L. Olmsted, L.G. Hector Jr, Nat. Mater. 5 (2006) 875-880.

[20] M. Legros, G. Dehm, E. Arzt, T.J. Balk, Science 319 (2008) 1646-1649.

[21] A.M. Hunt, D.W. Pashley, J. Phys. Radium 23 (1962) 846-853. 
[22] B.A. Greenberg, G. Hug, O.V. Antonova, T.S. Boyarshinova, Z.M. Pesina, I.N. Sachanskaya, A.Yu. Volkov, Intermetallics 5 (1997) 297-309.

[23] A.Yu. Volkov, V.A. Kazantsev, Phys. Met. Metallogr. 113 (2012) 62-71.

[24] N. Schell, A. King, F. Beckmann, T. Fischer, M. Müller, A. Schreyer, Mater. Sci. Forum (2014).

[25] Dassault Systèmes, Providence, RI, USA, (2011).

[26] J. Filik, A.W. Ashton, P.C.Y. Chang, P.A. Chater, S.J. Day, M. Drakopoulos, M.W. Gerring, M.L. Hart, O.V. Magdysyuk, S. Michalik, A. Smith, C.C. Tang, N.J. Terrill, M.T. Wharmby, H. Wilhelm, J. Appl. Crystallogr. 50 (2017) 959-966.

[27] S. Hashimoto, Acta Crystallogr. A 30 (1974) 792-798.

[28] S. Hashimoto, Acta Crystallogr. A 37 (1981) 511-516.

[29] S. Hashimoto, Acta Crystallogr A 39 (1983) 524-530.

[30] T. Anraku, I. Sakaihara, T. Hoshikawa, M. Taniwaki, Mater. Trans. 50 (2009) 683-688. 